\section{Can End-tidal Carbon Dioxide Levels Be Used for Determining Tissue Oxygen Saturation in Smokers and Nonsmokers?}

\author{
Sadiye Yolcu ${ }^{1}$, Adem Kaya ${ }^{1}$
}

${ }^{1}$ Adana City Research and Education Hospital Department of Emergency Medicine, Adana, Turkey

\section{Correspondence:}

Sadiye Yolcu, Assoc Prof.

Address: Adana Sehir Hastanesi Acil Tip Klinigi, Adana, Turkey

Email: sadiyeyolcu@yahoo.com
Received: 24.02.2019,

Accepted: 19.03.2019

https://doi.org/10.5799/jcei/5759

\begin{abstract}
Objectives: End-tidal carbon dioxide (EtCO2) monitoring has been used in a range areas, such as invasive sedation procedures, during cardiopulmonary resuscitation, ventilation monitoring of patients with altered mental status, trauma and respiratory system diseases in emergency departments. Near-infrared spectroscopy (NIRS) allows continuous, noninvasive measurement of tissue hemoglobin oxygen saturation (StO2) in muscle and has been studied for a wide range of conditions.

In this study we aim to determine the levels of $\mathrm{EtCO} 2$ and $\mathrm{StO} 2$ in smokers and nonsmokers and the relationship between these two parameters.

Methods: We examined 201 healthy volunteers including 156 smokers and 45 nonsmokers. We measured thenar muscle StO2 via NIRS device. The EtCO2 was determined by a capnograph. Baseline measurements were obtained from all participants after not-smoking for two h. Second measurements were taken $5 \mathrm{~min}$ after smoking was finished.

Results: The mean baseline EtCO2 of the smoker group was 42.61 $\pm 4,94$ (min:31, max:54) $\mathrm{mmHg}$ and mean baseline StO2 for this group was $77.81 \pm 4.71 \%$ (min:69, max:86). In smokers, mean baseline EtCO2 (p:0.00), baseline StO2 (p:0.001), fifth min EtCO2 (p:0.00), fifth min StO2 ( $p: 0.02)$ levels, and pack*year ( $p: 0.00)$ were significantly different between males and females. In nonsmokers baseline EtCO2 and baseline StO2 levels were correlated (p:0.035,r:0.315). But we couldn't find significance between females and males for mean baseline EtCO2 (p:0,246) and baseline StO2 (p:0.264) levels in this group.
\end{abstract}

Conclusion: Smoking affects tissue oxygenation. In nonsmokers baseline EtCO2 and baseline STO2 levels were correlated, but there was no correlation for these two parameters in smokers. Clinicians may use EtCO2 levels for predicting the tissue oxygenation in nonsmoker patients.

Keywords: end-tidal carbon dioxide, near-infrared spectroscopy, tissue hemoglobin oxygen saturation, smoker, nonsmoker

\section{INTRODUCTION}

Capnograph was originally used for validation of endotracheal tube placement during cardiopulmonary resuscitation (CPR) studies in emergency practice $[1,2]$. But during recent years, end-tidal carbon dioxide (EtCO2) monitoring via capnograph has been used in a range of areas such as invasive sedation procedures, cardiopulmonary resuscitation effectivity, ventilation monitoring of patients with altered mental status, trauma and respiratory system diseases [3-7].
}

Near-infrared spectroscopy (NIRS) allows continuous, noninvasive measurement of tissue hemoglobin oxygen saturation (StO2) in muscle and has been studied in a wide range of conditions [8]. NIRS has been used in critically ill patients especially in intensive care units (ICUs) and also researches have still been studying the efficiacy and areas of use of this device [9]. It has also been used to classify the severity of hemorrhagic shock, as a guide for fluid resuscitation, and during elective surgery septic shock, multiple- organ dysfunction 
EtCO2 and StO2 in Smokers

\begin{tabular}{|c|c|c|c|c|c|}
\hline & \multicolumn{2}{|c|}{ Smoker Group } & \multicolumn{2}{|c|}{ Nonsmoker group } & \multirow[t]{2}{*}{$\mathbf{p}$} \\
\hline & $\mathbf{F}$ & $M$ & $\mathbf{F}$ & $M$ & \\
\hline \multirow{3}{*}{ Age } & $30.94 \pm 8.0$ & $34.06 \pm 10.58$ & $36.12 \pm 10.9$ & $37,61 \pm 16.74$ & \\
\hline & $(\min : 18, \max : 52)$ & $(\min : 18, \max : 67)$ & $(\min : 23, \max : 49)$ & $(\min : 24, \max : 71)$ & \\
\hline & \multicolumn{2}{|c|}{$\begin{array}{l}\text { Total: } 33.02 \pm 9.88 \\
\text { (min:18,max:67) }\end{array}$} & \multicolumn{2}{|c|}{$\begin{array}{l}\text { Total: } 37.04 \pm 17.72 \\
(\min : 23, \max : 71)\end{array}$} & 0.55 \\
\hline \multirow{3}{*}{$\begin{array}{l}\text { Baseline } \\
\text { EtCO2 } \\
\text { (mmHg) }\end{array}$} & $39.79 \pm 3.53$ & $44.02 \pm 4.94$ & $44.06 \pm 3.24$ & $43.11 \pm 4.71$ & \\
\hline & $(\min : 31, \max : 45)$ & $(\min : 35, \max : 54)$ & (min:38,max:50) & $(\min : 36, \max : 54)$ & \\
\hline & \multicolumn{2}{|c|}{$\begin{array}{l}\text { Total: } 42.61 \pm 4.94 \\
(\min : 31, \max : 54)\end{array}$} & \multicolumn{2}{|c|}{$\begin{array}{l}\text { Total: } 43.47 \pm 4.21 \\
(\min : 36, \max : 54)\end{array}$} & 0.195 \\
\hline \multirow{2}{*}{$\begin{array}{c}\text { Baseline } \\
\text { StO2 } \\
(\%)\end{array}$} & $\begin{array}{c}75.96 \pm 5.23 \\
(\min : 69, \max : 86)\end{array}$ & $\begin{array}{c}78.73 \pm 4.16 \\
(\min : 70, \max : 86)\end{array}$ & $\begin{array}{c}75.71 \pm 5.58 \\
(\min : 65, \max : 81)\end{array}$ & $\begin{array}{c}75.32 \pm 2.85 \\
(\min : 70, \max : 80)\end{array}$ & \\
\hline & \multicolumn{2}{|c|}{$\begin{array}{l}\text { Total: } 77.81 \pm 4.71 \\
\text { (min:69,max:86) }\end{array}$} & \multicolumn{2}{|c|}{$\begin{array}{l}\text { Total: } 75.47 \pm 4.05 \\
\text { (min:65,max:81) }\end{array}$} & 0.004 \\
\hline
\end{tabular}

syndrome and as a vital sign prehospitally [10-16].

In this study we aim to determine the levels of EtCO2 and $\mathrm{StO} 2$ in smokers and nonsmokers and the relationship between these two parameters and besides whether EtCO2 levels may help clinicans to predict the $\mathrm{StO} 2$ levels in smoker and nonsmoker patients.

\section{METHOD}

\section{Subjects}

We examined 201 healthy volunteers: 156 smokers and 45 nonsmokers. Participants with chronic or acute diseases, history of drug usage, pregnant women and volunteers under 18 years of age were excluded from the study.

\section{$\mathrm{StO} 2$}

In our study, we measured thenar muscle StO2 via widegap second-derivative NIRS (InSpectra; Hutchinson Technology). This device serves to predict the hemoglobin Sto 2 in the microvasculature of muscle tissue, including the arteriolar, capillary and venular compartments [17].

\section{EtCO2}

EtCO2 was determined by the EMMA capnograph (Masimo Sweden AB), which measures end-tidal pressure by means of an infrared-absorbing sensor. We also used an EMMA airway adaptor and 7.0 endotracheal tube to evaluate the expiratuar breathing.

\section{Experimental Protocol}

Our study was approved by the Human Study Committee of our medical faculty (10.04.2014/43). The volunteers were informed and gave their informed consent. All measurements took place in a temperature-controlled environment. The study group was comfortably seated and breathing normally before, during and after the measurement. Smokers had been instructed to refrain from smoking for at least $2 \mathrm{~h}$ prior to the measurements. Baseline measurements were obtained from all participants. Smokers were then asked to smoke a cigarette according to their habitual brand, maintaining the usual aspiration pattern. Second measurements were taken 5 min after smoking was finished. Each participant held the endotracheal tube placed to the airway adapter of the capnograph in his or her mouth and expirated for 5 seconds. The last values on the screen were recorded before and after smoking. Similarly, for the StO2 levels, an Inspectra device was placed to the right thenar muscle for $10 \mathrm{~s}$ and mean of the first, fifth and 10th second values were noted.

\section{Statistical Analysis}

The normal distribution and homogeneity of each parameter were tested using the Shapiro- Wilk test and the Kolmogorov-Smirnov test. Age, gender, baseline ETCO2, baseline STO2, fifth min Etco2 and fifth min StO2 values did not suit the normal distribution. A Mann Whitney-U test was used for differences between and among two groups. A Spearman correlation test was used for correlation analysis. In all tests the significance level was $\mathrm{p}<0.05$. SPSS (Statistical Package for the Social Sciences) software 18.0 was used for allanalysis.

\section{RESULTS}

Our study included 156 smokers (77.6\%) and 45 nonsmokers (22.4\%), a total of 201 healthy volunteers. Mean age of the smoker group was 33.02 \pm 9.88 (min:18, max:67). The mean baseline ETCO2 of the smoker group was 42.61 $\pm 4,94$ (min:31, max:54) $\mathrm{mmHg}$, and the mean baseline STO2 for this group was $77.81 \pm 4.71 \%$ (min:69, max:86). Age, baseline ETCO2, baseline STO2, and cigarette pack ${ }^{*}$ year distributions of the groups according to gender are detailed in Table 1. The mean fifth min EtCO2 of the smoker group was $47.11 \pm 5.33$ (min:33, max:57) $\mathrm{mmHg}$ and the mean fifth min StO2 of the smoker group was $81.71 \pm 4.73 \%$ (min:73, max:91). The mean cigarette pack ${ }^{\star}$ year was $14.24 \pm 14.88$ (min:1, max:80) in the smoker group. In the smoker group, mean baseline ETCO2 (p:0.00), baseline STO2 (p:0.001), fifth min ETCO2 (p:0.00), fifth min STO2 (p:0.02) levels, and 
$\operatorname{pack}^{\star}$ year (p:0.00) were significantly different between males and females (Table 1).

In the smoker group the mean second ETCO2 level was 43.77 \pm 4.373 (min:33, max:50) $\mathrm{mmHg}$, mean second $\mathrm{StO} 2$ level was $80.29 \pm 5.17 \%$ (min:73, max:88) and mean pack $^{*}$ year was $8.71 \pm 7.59$ (min:1, max:30) in females and; these values for males were $48.78 \pm 4.99 \mathrm{mmHg}$ ( $\min : 36$, max:57) $\mathrm{mmHg}$, $82.42 \pm 4.35 \%$ (min:74, max:91), and 17.01 \pm 16.77 (min:1, max:80) respectively.

In nonsmokers baseline EtCO2 and baseline StO2 levels were correlated (p:0.035, r:0.315). But we couldn't find significance between females and males for mean baseline EtCO2 (p:0.246) and baseline StO2 (p:0.264) levels in this group.

Age was correlated with baseline StO2 (p:0.00,r:0.29) but there was no correlation with baseline EtCO2 (p:0.216, r:0.08). In the smokers group, baseline EtCO 2 was correlated with fifth $\min$ EtCO2 (p:0.00, r:0.75) and pack ${ }^{\star}$ year (p:0.003,r:0.23) but we couldn't determine a correlation with baseline StO2 (p:0,880, r:-0.012) and fifth min StO2 (p:0.47, $\mathrm{r}: 0.57)$. Similarly baseline $\mathrm{StO} 2$ was correlated with fifth min StO2 (p:0.00,0.84) and pack ${ }^{\star}$ year (p:0.00, r:0.30). Pack ${ }^{\star}$ year was also positively correlated with fifth min EtCO2 (p:0.003, r:0.23) and fifth min STO2 (p:0.001, r:0.26).

\section{DISCUSSION}

In our study, baseline STO2 levels of smokers were higher than those of nonsmokers, but ETCO2 values didn't differ. In nonsmokers baseline EtCO2 and baseline StO2 levels were correlated. But there was no correlation for these two parameters in smokers.

In the literature, there have been several studies on the use of emergency EtCO2 monitoring. Wahlen et al's study investigated EtCO2 values in spontaneously breathing patients. They searched for the influence of EtCO2 monitoring on therapeutic decisions by emergency physicians. Their study included 299 patients were included and reported pathological EtCO2 values in 19 patients (6.3\%). They suggested that EtCO2 monitoring may be a useful additional variable in spontaneously breathing patients [17].

Another study examined the relationship among EtCO2 and other oxygenation markers such as arterial oxygen pressure $(\mathrm{PaO} 2)$, carbon dioxide pressure $(\mathrm{PaCO} 2)$, and pulse oximetry (SpO2). Giner performed this study in 57 active smokers. In this study, the mean $\mathrm{SpO} 2$ of the participants was 95\%, and oxygen saturation measured directly in arterial blood samples was $95.1 \%$. The mean EtCO2 was $37.9 \mathrm{~mm} \mathrm{Hg}$ and $\mathrm{PaCO} 2$ by arterial blood gas analysis was $40.6 \mathrm{~mm} \mathrm{Hg}$. They found a correlation between EtCO2-SpO2 and EtCO2-PaCO2. Although this suggested that both oxyhemoglobin saturation assessments and both $\mathrm{CO} 2$ pressure determinations were closely related, it cannot be inferred that the 2 techniques in each case are completely congruent. Pulse oximetry and capnography are both useful for monitoring oxyhemoglobin saturation or ventilation [18].

In a recent study, $\mathrm{StO} 2$ monitoring for acute effects of smoking has been reported by Siafaka et al. Similarly to our study, they used NIRS to compare the microcirculatory function of smokers with that of nonsmokers. They studied 25 smokers and 40 nonsmokers, but their study protocol was different from ours except for its measurement times and measurement area (thenar region). They asked the participants not to smoke for $2 \mathrm{~h}$ beforel the baseline measurement and took the last measurement after $5 \mathrm{~min}$ too. But they preferred a continious $\mathrm{StO} 2$ monitoring. They recorded $\mathrm{StO} 2$ baseline values for $5 \mathrm{~min}$. Subsequently, the brachial artery occlusion technique was applied to evaluate microcirculatory function before, during, and after smoking a cigarette. In that study, StO2 before smoking was 85 , which did not differ significantly between genders and StO2 did not change significantly during smoking. $\mathrm{O} 2$ consumption rate was significantly greater in women at baseline and throughout the smoking session. $\mathrm{O} 2$ consumption rate was reduced during smoking and at $5 \mathrm{~min}$ after the smoking session. Smoking had a significant effect on vascular reactivity, with no significant differences between genders. Five minutes after smoking, vascular reactivity had returned to approximately normal levels [19].

In another study, Terborg et al searched for cerebral blood flow and oxygenation in 24 healthy volunteers via transcranial Doppler sonography and near-infrared spectroscopy before, during, and after smoking a cigarette. They simultaneously recorded cerebral blood flow velocity of both middle cerebral arteries, mean arterial blood pressure, skin blood flow, EtCO2, changes in concentration of cerebral oxyhemoglobin, deoxyhemoglobin, and total hemoglobin, and a cerebral tissue oxygenation index. Smoking increased cerebral blood flow velocity, oxyhemoglobin, and total hemoglobin. After smoking, the increase in cerebral blood flow velocity and total hemoglobin persisted, while oxyhemoglobin returned to baseline. Deoxyhemoglobin and cerebral tissue oxygenation index did not change during the whole procedure. During smoking, but not after, cerebral blood flow velocity increase was correlated to ipsilateral changes in oxyhemoglobin and total hemoglobin. In this study, tissue oxygenation index was $64.6 \%$ before smoking, $65.5 \%$ during smoking, and $64.6 \%$ after smoking. Mean EtCO2 was $41.4 \mathrm{mmHg}$ and $40.2 \mathrm{mmHg}$ after smoking [20].

Akca et al examined brain tissue oxygenation and EtCO2 monitoring in 20 surgical patients to determine the postoperative infection risk. In this study, the patients underwent intraoperative EtCO2, tissue oxygen partial pressure, transcutaneous oxygen tension and cerebral oxygen saturation monitoring via cerebral oximeter. The authors reported that mild intraoperative hypercapnia increased subcutaneous and cerebral oxygenation [21]. 


\section{CONCLUSION}

In our study, baseline $\mathrm{StO} 2$ levels of smokers were higher than those of nonsmokers, but EtCO2 values did not differ. As a result of this, we can say that smoking affects tissue oxygenation. In nonsmokers, baseline $\mathrm{EtCO} 2$ and baseline $\mathrm{StO} 2$ levels were correlated, but there was no correlation for these two parameters in smokers. EtCO2 measurement devices are much more easy to reach and cheaper when compared with near infrared spectroscope both in emergency departments and intensive care units. These results indicate that $\mathrm{EtCO} 2$ levels of nonsmoker patients may help clinicans to predict the $\mathrm{StO} 2$ levels.

Because of lack of literature, further comprehensive studies are needed to explain the reasons for these changes in acute and long term smoking the use of these parameters especially the $\mathrm{StO} 2$ in the ED.

\section{ACKNOWLEDGEMENTS}

S.Y. and A.K. have made substantial contributions to conception and design, or acquisition of data, or analysis and interpretation of data, has drafted the submitted article or revised it critically for important intellectual content; has provided final approval of the version to be published; has agreed to be accountable for all aspects of the work in ensuring that questions related to the accuracy or integrity of any part of the work are appropriately investigated and resolved.

Declaration of interest: The authors report no conflicts of interest.

Financial Disclosure: No financial support was received.

\section{REFERENCES}

1. Sanders AB, Atlas M, Ewy GA. Expired PCO2 as an index of coronary perfusion pressure. Am J Emerg Med 1985;3(2):147-9.

2. Sanders AB. Capnometry in emergency medicine. Ann Emerg Med 1989;18(12):1287-90.

3. Krauss B, Hess DR. Capnography for procedural sedation and analgesia in the emergency department. Ann Emerg Med 2007;50(2):172-81.

4. Neumar RW, Otto CW, Link MS. Part 8: adult advanced cardiovascular life support: 2010 American Heart Association Guidelines for Cardiopulmonary Resuscitation and Emergency Cardiovascular Care. Circulation 2010;122(18 Suppl 3):729-67.

5. Davis DP, Patel RJ. Noninvasive capnometry for continuous monitoring of mental status: a tale of 2 patients. Am J Emerg Med 2006;24(6):752-4.

6. Corbo J, Bijur P, Lahn M. Concordance between capnography and arterial blood gas measurements of carbon dioxide in acute asthma. Ann Emerg Med 2005;46(4):323-7.
7. Helm M, Schuster R, Hauke J. Tight control of prehospital ventilation by capnography in major trauma victims. Br J Anaesth 2003;90(3):327-32.

8. Mancini DM, Bolinger L, Hao L. Validation of nearinfrared spectroscopy in humans. J Appl Physiol. 1994;77(6):2740 -2747.

9. Crookes BA, Cohn SM, Bloch S. Can near-infrared spectroscopy identify the severity of shock in trauma patients? J Trauma 2005;58(4):806. discussion 813.

10. Moore FA, Nelson T, McKinley BA. Massive transfusion in trauma patients: tissue hemoglobin oxygen saturation predicts poor outcome. J Trauma 2008;64(4):1010-23.

11. George ME, Beilman GJ, Mulier KE. Noninvasive tissue oxygen saturation measurements identify supply dependency. J Surg Res 2010;160(1):40-6.

12. Cohn SM, Pearl RG, Acosta SM, et al. A prospective randomized pilot study of near infrared spectroscopy directed restricted fluid therapy versus standard fluid therapy in patients undergoing elective colorectal surgery. Am Surg 2010;76(12):1384-92.

13. Rodriguez A, Lisboa T, Martin-Loeches I. Mortality and regional oxygen saturation index in septic shock patients: a pilot study. J Trauma 2011;70(5):1145-52.

14. Cairns CB, Moore FA, Haenel JB. Evidence for early supply independent mitochondrial dysfunction in patients developing multiple organ failure after trauma. J Trauma. 1997;42(3):532-6.

15. Sagraves SG, Newell MA, Bard MR. Tissue Oxygenation Monitoring in the Field: A New EMS Vital Sign. J Trauma. 2009;67(3): 441-3.

16. Myers DE, Anderson LD, Seifert RP. Noninvasive method for measuring local hemoglobin oxygen saturation in tissue using wide gap second derivative near-infrared spectroscopy. J Biomed Optics 2005; 10(3):034017.

17. Wahlen BM, Bey T, Wolke BB. Measurement of endtidal carbon dioxide in spontaneously breathing patients in the pre-hospital setting. A prospective evaluation of 350 patients Resuscitation.2003;56(1):35-40.

18. Giner J, Casan P. Pulse Oximetry and Capnography in Lung Function Laboratories. Arch Bronconeumol 2004;40(7):311-4.

19. Siafaka A, Angelopoulos E, Kritikos K. Acute Effects of Smoking on Skeletal Muscle Microcirculation Monitored by Near-Infrared Spectroscopy. Chest.2007;131(5):1479-85.

20. Terborg C, Birkner T, Schack B, et al. Acute effects of cigarette smoking on cerebral oxygenation and hemodynamics: A combined study with near-infrared spectroscopy and transcranial Doppler sonography. J Neurol Sci.2002;205(1):71- 5.

21. Akca O, Liem E, Suleman MI. Effect of intra-operative end-tidal carbon dioxide partial pressure on tissue oxygenation. Anaesthesia, 2003;58(6):536-42. 